\title{
Estilo de vida en adultos jóvenes universitarios de Barranquilla, Colombia. Diferencias según sexo y estatus socioeconómico Lifestyle of university students from Barranquilla, Colombia. Differences according sex and socioeconomic status
}

*Yisel Pinillos-Patiño; **Roberto Rebolledo-Cobos; *Yaneth Herazo-Beltrán; *Enny Oviedo-Argumedo; *Mario Guerrero Ospino; *Patricia Valencia-Fontalvo; ***Gabriela Cortés-Moreno

*Universidad Simón Bolívar (Colombia), **Universidad Metropolitana (Colombia)***Dirección General de Calidad y Educación en Salud (México)

Resumen. El estilo de vida es concebido como un comportamiento cotidiano y que permanece en el tiempo, el cual evidencia los patrones individuales de conducta de una persona; además, y está determinado por factores sociales, culturales y personales. La etapa universitaria se constituye en este sentido, en una oportunidad para promover la modificación de hábitos por conductas saludables. El objetivo del estudio fue determinar los estilos de vida en estudiantes universitarios y las diferencias según el sexo y el estatus socioeconómico. Se realizó un estudio cuantitativo, descriptivo de corte transversal con 508 universitarios a quienes se les aplicó una encuesta auto administrada sobre sus condiciones sociodemográficas, mientras que los estilos de vida fueron evaluados mediante el cuestionario de Perfil del Estilo deVida de Pender II (PEVP-II). Se observó que solo el 24,02\% (n=122) de la muestra controla frecuentemente los niveles de azúcares y de grasas el 21,85\% ( $n=111)$, en su alimentación; más del 20\% ( $n=102)$ no realiza actividad física, entre el $20(n=102)$ y 30\% (n=152) no regulan los azúcares o incluyen frutas y vegetales en sus dietas; los promedios inferiores al 60\%(n=305) en los factores asociados contemplados en el estudio, infieren que los encuestados no mantienen un estilo de vida saludable. En el período de la vida universitaria, el estilo de vida está influenciado por cambios de comportamientos asociados a la formación personal, al entorno familiar y al contexto cultural y social en que desarrolla cada individuo. Los bajos niveles de práctica de actividad física y la presencia de hábitos considerados riesgosos en la dimensión nutricional, pueden estar relacionados con los estilos de vida, y propiciar el desarrollo de enfermedades no transmisibles que conllevan a incrementar la morbilidad y mortalidad en las etapas del curso de la vida del ser humano.

Palabras Clave: actividad física, estilo de vida saludable, estado nutricional, factores de riesgo, adultos jóvenes, universidades (DeCs).

\begin{abstract}
Lifestyles are everyday behaviors that show an individual's way of life and are usually maintained over time. The university stage is an important period to establish healthy habits for the rest of life. The objective of the study was to determine the lifestyles of university students and the differences according to sex and socioeconomic status. A cross-sectional study was carried out in 508 university students to whom a self-administered survey was applied on their sociodemographic conditions, while their lifestyles were evaluated using the PEVP-II questionnaire. It was observed that only $24.02 \%$ of the sample frequently controlled the levels of sugars and fats, $21.85 \%$, in their diet; more than $20 \%$ do not perform physical activity, between 20 and 30\% do not regulate sugars or include fruits and vegetables in their diets; the averages lower than $60 \%$ in the associated factors contemplated in the study, infer that the respondents do not maintain a healthy lifestyle. Finally, it is concluded that low levels of physical activity practice and the presence of habits considered risky in the nutritional dimension, may be related to lifestyles, making this identification in the university environment can help decision-making for design of intervention strategies supported in the wellbeing of students.
\end{abstract}

Key words: motor activity. Healthy life style, nutritional status, risk factors, young adults, universities (MeSH).

\section{Introducción}

Los estilos de vida representan los hábitos que permiten identificar el modo de vida de una persona, desde los aspectos psicológicos, económicos, culturales y sociales, factores que, al conjugarse con el ingreso al ambiente universitario, representa un papel preponderante en el desarrollo personal del individuo (Oliveros, Benítez, García \& Bello, 2016; Canova, Quintana \& Álvarez, 2018). Esta etapa que va acompañada de variedad de cambios emocionales, fisiológicos, ambientales,

Fecha recepción: 22-01-21. Fecha de aceptación: 14-09-21

Yisel Pinillos Patiño

ypinillos@unisimonbolivar.edu.co educativos, alimentarios y familiares pueden o no asociarse a una serie de comportamientos de riesgo (Chau \& Tavera, 2020) según sea la elección de cada persona de acuerdo con sus gustos y preferencias para el desarrollo de actividades en su tiempo libre, para el manejo del estrés, la selección de los alimentos a consumir, entre otros, momento para afianzar e instaurar conductas y hábitos saludables para el resto de la vida (Durán, et al., 2017). Además, es una etapa de transición al mundo laboral, por lo que se le considera como un puente entre la adolescencia y la edad adulta (Chau \& Tavera, 2020).

Por su parte la Organización Mundial de la Salud (OMS, 2018), devela que el consumo de tabaco, la inactividad física, el uso nocivo del alcohol y las dietas mal- 
sanas aumentan el riesgo de morir por causa de las Enfermedades No Transmisibles (ENT), las cuales ocasionan 41 millones de muertes cada año (71\%) con relación a las presentadas en el mundo, con mayor frecuencia las enfermedades cardiovasculares (17,9 millones por año), seguidas del cáncer (9,0 millones), las enfermedades respiratorias (3,9 millones) y la diabetes $(1,6$ millones).

La obesidad como factor de riesgo, es un evento que afecta la salud y que va en aumento, cerca del 56,5\% de los adultos tiene exceso de peso, de estos el 18,7\% tienen obesidad y 37,8\% tienen sobrepeso (Minsalud, 2015). La población universitaria, se encuentra expuesta a factores en sus actividades diarias condicionadas por horarios discontinuos, tiempo prolongado frente a pantallas, conductas alimentarias de riesgo, adicciones y violencia social, lo que en conjunto puede llegar a aumentar la probabilidad del riesgo para la salud (Espinoza \& Vanegas, 2017).

Todo este recorrido nos permite afirmar que las acciones en materia de promoción de la salud deben invitar a las personas a tener un mayor control de su propio bienestar, y tal como lo recomienda la OMS, englobar una gama de intervenciones sociales y ambientales enfocadas a beneficiar y proteger la salud y la calidad de vida individual mediante la prevención y solución de las causas esenciales de los problemas de salud, y no centrándose únicamente en el tratamiento y la curación (OMS, 2018).

$\mathrm{Al}$ considerar que los estudiantes en el ámbito universitario pasan gran parte de su tiempo diario en la institución educativa, y bajo la premisa de que la formación profesional propende por el desarrollo holístico del discente, sería importante, al mismo tiempo que se identifican las conductas de riesgo que afectan la salud y favorecen el desarrollo de ENT en esta etapa de la vida, incluir en su formación académica contenidos y espacios que fomenten el autocuidado y hábitos vida más saludables; ello, además de generar bienestar físico, contribuirá al mantenimiento general de la salud, así como al fomento de una cultura de salud en la comunidad educativa (OMS, 2018).

Diversos estudios han identificado los factores asociados a los estilos de vida en los jóvenes en la etapa universitaria, tales como los socioculturales y las características personales, las actitudes, hábitos, conductas, decisiones y valores, que afectan positiva o negativamente su salud creando un riesgo autoimpuesto que consecuentemente le induce a enfermar o incrementa la posibilidad de morir por múltiples causas, entre las cuales se encuentra la enfermedad cardiovascular relacionada con el consumo de alcohol y tabaco (Gómez, Landeros, Romero \& Troyo, 2016; Suescún et al., 2017). Entre tanto, un estilo de vida físicamente activo, entendido este como un nivel de actividad física moderado/ alto, ha mostrado favorecer la mejoría del valor global de los hábitos de vida en grupos de jóvenes universitarios; es decir, una mejor percepción de calidad de vida global y en salud, al igual que en los dominios físico, psicológico y medio ambiente, comparado con estudiantes físicamente inactivos (Pérez, Rivera \& Delgado, 2017; Concha, Castillo \& Guzmán, 2019).

Lo anterior, permite inferir sobre la efectividad de estrategias enmarcadas en la promoción de estilos de vida físicamente activos y saludables; además, deja entrever la necesidad de diseñar, formular e implementar estrategias educativas que promuevan la participación activa del estudiantado a fin de partir de los intereses, expectativas y necesidades sentidas que expresen los actores del contexto universitario, y de esta manera los programas, políticas y estrategias sean sostenibles en el tiempo y con beneficios observables en el bienestar de los jóvenes. El propósito de este estudio fue determinar los estilos de vida en estudiantes universitarios y las diferencias según el sexo y el estatus socioeconómico

\section{Materiales y método}

Diseño del estudio: Se realizó un estudio cuantitativo, descriptivo de corte transversal, en una Institución de Educación Superior (IES) privada en la ciudad de Barranquilla, Colombia, en el cual midió el resultado, los estilos de vida, y las exposiciones, las variables sociodemográficas sexo y estatus sociodemográfico, al mismo tiempo. Los participantes fueron seleccionados en función de los criterios de inclusión y de exclusión establecidos para el estudio (Setia, 2016).

Población y Muestra: de un total de 14623 estudiantes matriculados en los 18 programas académicos de pregrado de las cinco facultades con las que cuenta la universidad participante, se calculó una muestra de 554 estudiantes matriculados en el período 2019-2, teniendo en cuenta un 95\% de confianza, un error del 4\% y una frecuencia esperada del 40\%; al final se obtuvo un porcentaje de respuesta del 91,7\% que corresponde a 508 estudiantes. La selección se realizó mediante un muestreo aleatorio por conglomerados de dos etapas, en el cual la facultad o área de conocimiento fue considerada como la primera etapa y el programa académico la segunda etapa. Para tener una muestra represen- 
tativa de cada facultad, la distribución fue proporcional al número total de estudiantes matriculados en cada campo de conocimiento: Ciencias de la Salud (40\%), Ciencias Sociales (26\%), Ingenierías y Arquitectura (18\%), Economía y Finanzas (16\%).

Como criterio de inclusión para la selección de la muestra se consideró a estudiantes de todas las edades con matrícula activa en el período académico en que se realizó la recolección de la información y aceptar participar voluntariamente en el estudio. Se excluyeron a los estudiantes de posgrado.

Procedimiento: La información fue recolectada mediante la aplicación de una encuesta auto administrada con preguntas estructuradas y cerradas acerca de variables sociodemográficas entre las cuales se contemplaron el sexo, la edad, el estrato socioeconómico (referido este según el Departamento Administrativo Nacional de Estadística - DANE - a la clasificación en estratos de los inmuebles residenciales que deben recibir servicios públicos y se clasifica en: estrato 1 significa Bajo-bajo, el 2 es Bajo, 3 es Medio-bajo, 4 Medio, 5 Medio-alto y 6 Alto), el área residencial, el estado civil, la actividad laboral y la seguridad social. El estilo de vida fue evaluado con el cuestionario PEVP-II (Perfil del Estilo de Vida de Pender II), el cual fue validado por Walker y colaboradores (Espinosa, 2018) y mostró adecuadas propiedades para valorar las conductas promotoras de salud que constituyen un estilo de vida saludable; se evidenció un índice de consistencia interna de alfa de Cronbach de 0.94 para la escala completa, para la dimensión «manejo del estrés» un rango de 0.69 a 0.82 para las de «actividad física» y «crecimiento espiritual»; la subescala manejo del estrés un alfa de 0.68 , relaciones interpersonales 0.77 y responsabilidad de la salud 0.78. está integrado por 52 ítems agrupados en 6 dimensiones: nutrición, actividad física, manejo del estrés, relaciones interpersonales, crecimiento espiritual y responsabilidad de la salud, con opción de respuesta tipo Likert, con los siguientes criterios de puntuación: Nunca $(\mathrm{N})=1$ punto, alguna vez $(\mathrm{A})=2$ puntos, frecuentemente $(\mathrm{F})=3$ puntos y rutinariamente $(\mathrm{R})=4$ puntos. La puntuación máxima para el cuestionario general es de 208 puntos, mientras que, para las dimensiones Nutrición, Relaciones Interpersonales, Crecimiento Espiritual y Responsabilidad en Salud son 36 puntos y para las dimensiones Actividad Física y Manejo del Estrés de 32 puntos.

Consideraciones éticas: De acuerdo con el artículo 11 de la Resolución 8430/93 del Ministerio de Salud de la República de Colombia, la presente investigación es considerada sin riesgo. Previo a la aplicación de los instrumentos de evaluación, se solicitó la firma del consentimiento informado a cada participante mayor de 18 años de edad y el asentimiento informado para los menores de 18 años. La información recolectada en este estudio hizo parte del proyecto Factores Asociados a los Estilos de Vida en Estudiantes de Siete Universidades de la Región Caribe Colombiana avalado y financiado por el Departamento Administrativo de Ciencia, Tecnología e Innovación (Colciencias), denominado Ministerio de Ciencia, Tecnología e Innovación, a partir del año 2020 y contó con aprobación del comité de ética de la Universidad Simón Bolívar de Barranquilla. Los datos personales fueron tratados conforme con lo previsto en el Decreto 1377 de 2013 del Ministerio Comercio, Industria y Turismo (2013), reglamentario de la Ley 1581 de 2012 (Congreso de Colombia, 2012).

El procesamiento de los datos y análisis de la información se realizó mediante el paquete estadístico software SPSSâ versión 24.0 (Licencia de la Universidad Simón Bolívar). Las variables categóricas provenientes de la caracterización sociodemográfica y de las respuestas específicas del cuestionario son descritas mediante frecuencias absolutas y relativas, mientras que la puntuación derivada de la ponderación del cuestionario se expone en medidas de tendencia central.

Para determinar la normalidad entre los datos agrupados se aplicó la prueba de Kolmogorov-Smirnov, estableciendo así la bondad de ajuste de distribuciones con probabilidad entre las mismas. A razón de la distribución normal de todos los datos, las diferencias estadísticas entre las medias de las puntuaciones fueron determinados a través de la prueba ANOVA, determinando así las variaciones de las dimensiones que constituyen el Cuestionario PEVP-II entre grupos independientes, específicamente considerando como grupos al sexo y los diferentes estratos socioeconómicos.

\section{Resultados}

Las características sociodemográficas de los participantes evidenciaron mayor frecuencia del sexo femenino 62,80\% ( $\mathrm{n}=319)$; una media de edad de 20,74 $\pm 6,99$ años, para el rango de 18 y 22 años 79,52\% (n=404), seguidos de los participantes con edades entre 23 y 27 años $11,42 \%(n=58)$.

Los estudiantes participantes estaban adscritos con mayor frecuencia a los programas académicos de las ciencias de la salud, seguidos de los estudiantes de las ciencias sociales, ingenierías, arquitectura y del programa 


\begin{tabular}{|c|c|c|}
\hline Característica & Frecuencia (n) & $\%$ \\
\hline \multicolumn{3}{|c|}{ Sexo } \\
\hline Femenino & 319 & 62,80 \\
\hline Masculino & 189 & 37,20 \\
\hline \multicolumn{3}{|c|}{ Edad (años) } \\
\hline$<18$ & 26 & 5,12 \\
\hline $18-22$ & 404 & 79,52 \\
\hline $23-27$ & 58 & 11,42 \\
\hline $28-32$ & 9 & 1,77 \\
\hline$>32$ & 11 & 2,17 \\
\hline \multicolumn{3}{|c|}{ Programas académicos } \\
\hline Ciencias de la Salud & 207 & 40,75 \\
\hline Ciencias Sociales & 134 & 26,38 \\
\hline Ingenierías y Arquitectura & 90 & 17,71 \\
\hline Economía y Finanzas & 77 & 15,16 \\
\hline \multicolumn{3}{|c|}{ Estrato Socioeconómico } \\
\hline 1 & 158 & 31,10 \\
\hline 2 & 167 & 32,87 \\
\hline 3 & 143 & 28,15 \\
\hline 4 & 35 & 6,89 \\
\hline 5 & 2 & 0,39 \\
\hline 6 & 3 & 0,59 \\
\hline \multicolumn{3}{|c|}{ Estado Civil } \\
\hline Soltera (o) & 478 & 94,09 \\
\hline Casada (o) & 6 & 1,18 \\
\hline Unión Libre & 22 & 4,33 \\
\hline Viuda (o) & 2 & 0,39 \\
\hline \multicolumn{3}{|c|}{ Ocupación Laboral } \\
\hline No trabaja & 418 & 82,28 \\
\hline Si trabaja & 90 & 17,72 \\
\hline
\end{tabular}

de economía y finanzas. Más de la mitad de los estudiantes pertenecían a los estratos socioeconómicos 1 y 2, mientras que los pertenecientes a estrato 5 o 6 representaba menos del 1\%. La gran mayoría de los sujetos estudiados eran solteros $94,09 \%(n=478)$ y no se en-

\begin{tabular}{|c|c|c|}
\hline Pregunta & Frecuencia $(\mathrm{n})$ & $\%$ \\
\hline \multicolumn{3}{|c|}{ Escojo una dieta baja en grasas, grasas saturadas y en colesterol. } \\
\hline Nunca & 120 & 23,62 \\
\hline Algunas Veces & 277 & 54,53 \\
\hline Frecuentemente & 111 & 21,85 \\
\hline Rutinariamente & 0 & 0 \\
\hline \multicolumn{3}{|c|}{ Limito el uso de azucares y alimentos que contengan azúcar. } \\
\hline Nunca & 129 & 25,39 \\
\hline Algunas Veces & 257 & 50,59 \\
\hline Frecuentemente & 11 & 2,17 \\
\hline Rutinariamente & 111 & 21,85 \\
\hline \multicolumn{3}{|c|}{ Como de 6-11 porciones de pan, cereales, arroz, o pastas (fideos) todos los días. } \\
\hline Nunca & 102 & 20,08 \\
\hline Algunas Veces & 219 & 43,11 \\
\hline Frecuentemente & 15 & 2,95 \\
\hline Rutinariamente & 172 & 33,86 \\
\hline \multicolumn{3}{|c|}{ Como de 2 a 4 porciones de frutas todos los días } \\
\hline Nunca & 111 & 21,85 \\
\hline Algunas Veces & 295 & 58,07 \\
\hline Frecuentemente & 9 & 1,77 \\
\hline Rutinariamente & 93 & 18,31 \\
\hline \multicolumn{3}{|c|}{ Como de 3 a 5 porciones de vegetales todos los días. } \\
\hline Nunca & 125 & 24,61 \\
\hline Algunas Veces & 251 & 49,41 \\
\hline Frecuentemente & 26 & 5,12 \\
\hline Rutinariamente & 106 & 20,87 \\
\hline \multicolumn{3}{|c|}{ Como de 2 a 3 porciones de leche, yogurt, o queso cada día. } \\
\hline Nunca & 49 & 9,65 \\
\hline Algunas Veces & 266 & 52,36 \\
\hline Frecuentemente & 32 & 6,30 \\
\hline Rutinariamente & 161 & 31,69 \\
\hline \multicolumn{3}{|c|}{$\begin{array}{l}\text { Como solamente de } 2 \text { a } 3 \text { porciones de carnes, aves, pescado, frijoles, huevo y nueces todos lo } \\
\text { días. }\end{array}$} \\
\hline Nunca & 25 & 4,92 \\
\hline Algunas Veces & 192 & 37,80 \\
\hline Frecuentemente & 58 & 11,42 \\
\hline Rutinariamente & 233 & 45,87 \\
\hline \multicolumn{3}{|c|}{$\begin{array}{l}\text { Leo las etiquetas nutritivas para identificar el contenido de grasas y sodio en los alimentos } \\
\text { envasados. }\end{array}$} \\
\hline Nunca & 149 & 29,33 \\
\hline Algunas Veces & 211 & 41,54 \\
\hline Frecuentemente & 27 & 5,31 \\
\hline Rutinariamente & 121 & 23,82 \\
\hline \multicolumn{3}{|l|}{ Como y desayuno. } \\
\hline Nunca & 19 & 3,74 \\
\hline Algunas Veces & 134 & 26,38 \\
\hline Frecuentemente & 35 & 6,89 \\
\hline Rutinariamente & 320 & 62,99 \\
\hline
\end{tabular}

contraban vinculados a alguna actividad laboral 82,28\% $(\mathrm{n}=418) .($ Ver tabla 1$)$

En cuanto a la dimensión nutrición, se puede afirmar que el 24,02\% ( $n=122)$ de los sujetos elije de manera frecuente o incrusta en su rutina diaria dietas con control en la proporción de las grasas o de azucares. Del mismo modo, solo una quinta parte de la población afirma consumir de manera frecuente o rutinariamente frutas, además, una cuarta parte afirma consumir de 3 a 5 porciones de verduras diariamente. Más de 2 tercios de los sujetos no consideran revisar la etiqueta de los alimentos empacados antes de consumirlos y en casi la misma proporción, consideran desayunar cada día. (Ver tabla 2)

Los hallazgos relacionados con la dimensión actividad física, ninguno de los 508 individuos evaluados manifestó seguir un programa de ejercicios físicos de manera rutinaria, mientras que más de la cuarta parte de sujetos nunca realiza algún tipo de actividad física vigorosa por más de 20 minutos al menos tres veces por semana y así mismo, aproximadamente la quinta parte de los sujetos afirma no realizar en la misma frecuencia semanal ejercicios livianos. De manera general, más de la mitad de los sujetos estudiados consideran que no realizan suficiente actividad física o al menos con la su-

Tabla 3.

Frecuencias obtenidas en la dimensión actividad fisica

\begin{tabular}{|c|c|c|}
\hline Pregunta & Frecuencia $(\mathrm{n})$ & $\%$ \\
\hline \multicolumn{3}{|c|}{ Sigo un programa de ejercicios planificados } \\
\hline Nunca & 198 & 38,98 \\
\hline Algunas Veces & 201 & 39,57 \\
\hline Frecuentemente & 109 & 21,46 \\
\hline Rutinariamente & 0 & 0 \\
\hline \multicolumn{3}{|c|}{$\begin{array}{l}\text { Hago ejercicio vigoroso } 20 \text { o más minutos, por lo menos tres veces a la semana (tales como } \\
\text { caminar rápidamente, manejar bicicleta, baile aeróbico, usar la maquina escaladora). }\end{array}$} \\
\hline Nunca & 146 & 28,74 \\
\hline Algunas Veces & 211 & 41,54 \\
\hline Frecuentemente & 9 & 1,77 \\
\hline Rutinariamente & 142 & 27,95 \\
\hline \multirow{2}{*}{\multicolumn{3}{|c|}{$\begin{array}{l}\text { Tomo parte en actividades físicas livianas a moderadas (tales como caminar continuamente de } \\
30 \text { a } 40 \text { min, } 5 \text { o más veces a la semana). }\end{array}$}} \\
\hline & & \\
\hline Nunca & 109 & 21,46 \\
\hline Algunas Veces & 207 & 40,75 \\
\hline Frecuentemente & 14 & 2,76 \\
\hline Rutinariamente & 178 & 35,04 \\
\hline \multicolumn{3}{|c|}{ Como de 2 a 4 porciones de frutas todos los días. } \\
\hline Nunca & 102 & 20,08 \\
\hline Algunas Veces & 248 & 48,82 \\
\hline Frecuentemente & 31 & 6,10 \\
\hline Rutinariamente & 127 & 25 \\
\hline \multicolumn{3}{|c|}{ Hago ejercicios para estirar los músculos por lo menos 3 veces por semana. } \\
\hline Nunca & 127 & 25,00 \\
\hline Algunas Veces & 220 & 43,31 \\
\hline Frecuentemente & 29 & 5,71 \\
\hline Rutinariamente & 132 & 25,98 \\
\hline
\end{tabular}

Hago ejercicio durante actividades físicas usuales diariamente (tales como caminar a la hora del almuerzo, utilizar escaleras en vez de ascensor, estacionar el coche lejos del lugar de destino y caminar).

$\begin{array}{cc}\text { Nunca } & \\ \text { Algunas Veces } & 2 \\ \text { Frecuentemente } & \\ \text { Rutinariamente } & 1 \\ \text { Examino mi pulso cuando estoy haciendo ejercicio. } & \\ \text { Nunca } & 1 \\ \text { Algunas Veces } & 1 \\ \text { Frecuentemente } & \\ \text { Rutinariamente } & \\ \text { Alcanzo mi pulso cardiaco objetivo cuando hago ejercicio } \\ \text { Nunca } \\ \text { Algunas Veces } \\ \text { Frecuentemente } \\ \text { Rutinariamente }\end{array}$

\begin{tabular}{cc}
64 & 12,60 \\
218 & 42,91 \\
41 & 8,07 \\
185 & 36,42 \\
& \\
196 & 38,58 \\
186 & 36,61 \\
33 & 6,50 \\
93 & 18,31 \\
icio. & \\
105 & 20,67 \\
191 & 37,60 \\
32 & 6,30 \\
180 & 35,43 \\
\hline
\end{tabular}


ficiente frecuencia semanal en actividades cotidianas como caminar para trasportarse o subir escaleras. (Ver tabla 3)

La comparación de medias entre hombres y mujeres por dimensión estudiada permite observar que el promedio de resultados totales para hombres fue estadísticamente mayor en comparación con las mujeres $(\mathrm{p}<0,05)$, lo que significaría en general, un mejor estilo de vida en los primeros. Así mismo, las dimensiones manejo de estrés y actividad física mostraron un comportamiento similar, siendo estadísticamente mayores en los hombres que en las mujeres $(p<0,05)$. Las dimensiones relacionadas con el Comportamiento nutricional, Relaciones interpersonales, Crecimiento espiritual y Responsabilidad en salud no mostraron diferencias estadísticas entre sexos $(p>0,05)$. (Ver tabla 4)

Tabla 4.

\begin{tabular}{cccc}
\multicolumn{5}{c}{ Comparación de medias entre ambos sexos según las dimensiones estudiadas. } \\
\hline Dimensión & Hombres $(\mathrm{n}=189)$ & Mujeres $(\mathrm{n}=319)$ & P-valor \\
\hline Cuestionario General & $141,25 \pm 25,48$ & $135,87 \pm 22,93$ & 0,0145 \\
Nutricional & $22,87 \pm 5,02$ & $22,14 \pm 4,82$ & 0,1050 \\
Actividad Física & $20,95 \pm 5,97$ & $17,56 \pm 5,38$ & 0,0197 \\
Manejo del Estrés & $20,35 \pm 4,72$ & $19,34 \pm 4,56$ & 0,0468 \\
Relaciones Interpersonales & $25,62 \pm 4,96$ & $25,32 \pm 4,79$ & 0,4902 \\
Crecimiento Espiritual & $29,87 \pm 5,56$ & $29,33 \pm 5,18$ & 0,2664 \\
Responsabilidad en Salud & $22,00 \pm 6,09$ & $22,73 \pm 5,56$ & 0,1679 \\
\hline
\end{tabular}

Entre tanto que la comparación entre las medias obtenidas según la agrupación por estrato socioeconómico, por dimensión, y la cuantificación general promedio mostró que el promedio del resultado total del cuestionario PEVP-II de los sujetos de estrato 4 o mayor fue estadísticamente superior a cualquier otro grupo ( $\mathrm{p}<0,001)$, observando también una diferencia estadística entre el promedio de los sujetos estrato $1 \mathrm{y}$ estrato 3, siendo menor en los primeros. Con relación a la comparación individual de dimensiones, se puede observar que, en relación con la Actividad Física, el estrato 3 y 4 fue estadísticamente mayor al estrato 1 , así como también el estrato 4 o mayor y se diferenció del estrato 2 en esta dimensión. Finalmente, la dimensión Crecimiento Espiritual mostró que el estrato 4 o mayor tuvo el promedio más bajo, siendo estadísticamente inferior al resto de grupos. Las dimensiones Nutricional, Manejo del Estrés y Relaciones Interpersonales no mostraron diferencias estadísticas entre los grupos. (Ver tabla 5)

Tabla 5.

\begin{tabular}{cccccc}
\multicolumn{7}{c}{ Comparación de medias entre estratos socioeconómicos $y$ las dimensiones estudiadas. } \\
\hline \multicolumn{7}{c}{ Estrato socioeconómico } \\
Dimensión & $1(158)$ & $2(167)$ & $3(143)$ & $=4(40)$ & P-valor \\
\hline Cuestionario General & $135,25 \pm 23,40^{\mathrm{a}}$ & $138,44 \pm 24,26^{\mathrm{b} b}$ & $140,92 \pm 23,77^{\mathrm{b}}$ & $192,0 \pm 0,00^{\mathrm{c}}$ & $<0,000$ \\
Nutricional & $22,11 \pm 4,83$ & $22,38 \pm 4,91$ & $23,06 \pm 4,81$ & $21,4 \pm 5,34$ & 0,1836 \\
Actividad Física & $17,72 \pm 5,23^{\mathrm{a}}$ & $18,81 \pm 6,09^{\mathrm{ab}}$ & $19,50 \pm 5,96^{\mathrm{bc}}$ & $20,8 \pm 5,94^{\mathrm{c}}$ & 0,0066 \\
Manejo del Estrés & $19,73 \pm 4,48$ & $19,72 \pm 4,54$ & $19,89 \pm 4,66$ & $19,0 \pm 5,61$ & 0,7814 \\
Relaciones Interpersonales & $24,99 \pm 4,90$ & $25,60 \pm 4,76$ & $25,90 \pm 4,95$ & $24,8 \pm 4,68$ & 0,3136 \\
Crecimiento Espiritual & $29,35 \pm 5,50$ & $30,07 \pm 4,97$ & $29,85 \pm 4,98$ & $26,9 \pm 6,55^{\mathrm{a}}$ & 0,0055 \\
Responsabilidad en Salud & $21,97 \pm 5,49$ & $22,26 \pm 6,03$ & $23,25 \pm 5,66$ & $22,4 \pm 6,02$ & 0,2595 \\
\hline Datos que no comparten la misma letra en superíndice (a, bo o c) son estadísticamente diferentes.
\end{tabular}

\section{Discusión}

La etapa universitaria, se caracteriza por cambios profundos a nivel social, emocional, cultural e intelectual, al afrontar retos que requieren de la capacidad de adaptación a la realidad que ofrece el nuevo contexto social; así, los factores protectores permiten asegurar comportamientos que redundan en la salud física, mental y emocional de los estudiantes, los cuales, al mantenerse y consolidarse, afectan positivamente el resto de su vida con calidad (Herazo et al. 2020).

En este estudio se observó que la población pertenecía predominantemente a estratos socioeconómicos bajos y presentaba bajos niveles de práctica de actividad física; además, se evidenció hábitos de riesgo dentro de la dimensión de nutrición y sin asociación a una actividad laboral, factores que de cierta manera pueden ser asociados a estilos de vida con tendencias poco saludables manifestando así la importancia de detectarlos en el ambiente universitario a fin de reconocer las características que puedan estar alrededor de estos factores como posibles determinantes de los estilos de vida.

De acuerdo con los resultados descritos anteriormente y según Canova, Quintana \& Álvarez (2018), los estilos de vida están constituidos por diferentes aspectos o dimensiones, los cuales se van formando a lo largo de la historia de vida de los sujetos, tratándose de un proceso de aprendizaje familiar, social o de grupos formales de aprendizaje como la escuela o la universidad; estos pueden incidir positiva o negativamente sobre la calidad de vida de una persona; teniendo en cuenta que la entrada al ambiente universitario constituye un importante período de la vida, siendo una oportunidad inherente de desarrollo personal, es posible que esté asociada al desarrollo de comportamientos de riesgo. Estudios realizados previamente indican que los malos hábitos alimenticios, consumo de alcohol y cigarrillo y mal manejo del estrés son conexos (Bennasar, et al., 2020), el patrón de sueño irregular está asociado con el sobrepeso, la obesidad y con el bienestar psicosocial del individuo (Varmaghani et al., 2020) por lo cual la universidad debe intervenir en promoción de hábitos de vida saludable basado en las distintas condiciones de vida observadas en los jóvenes, de tal manera que se recurra a la integralidad de los abordajes colectivos (Gore, Menon, Safai, Shukla \& Yeravdekar, 2020).

Los resultados correspondientes a los hábitos alimentarios de este estudio se observa relación con lo reportado por De Souza, Zea, Rodríguez \& Molina (2017), en una muestra de jóvenes que el 61\% no in- 
cluía frutas ni verduras en su dieta diaria y el 57\% no evitaba el consumo elevado de grasas. Varela, Ochoa \& Tovar (2016) concuerda con este hábito reportado en los sujetos observados como un frecuente consumo de alimentos fritos, mecatos, dulces y gaseosas, aspecto que se correlaciona con los resultados de Canova (2017) y Villaquirán, Jácome \& Benavides (2018) respectivamente, quienes reportaron que sólo el 19\% de los estudiantes consumen frutas y verduras diariamente, y sólo el $38.1 \%$ de los participantes consumía frutas y verduras una vez por semana. En este sentido se pone en manifiesto que por distintos factores los jóvenes universitarios no cuidan la elección e ingesta de sus alimentos, combinado ello con otras dimensiones deficientes en sus estilos de vida, puede convertirse en un elemento clave para la aparición de ENT con impacto negativo en su calidad de vida.

En la evaluación de la dimensión actividad física, los sujetos reportaron no seguir de manera rutinaria un programa planificado de ejercicios, aspecto que concuerda con los reportes de otros autores que indican el hábito común en la población joven de una escasa o práctica nula de actividad física de por lo menos 30 minutos diarios o 150 minutos semanales, lo cual lleva a clasificarlos como un grupo de personas inactivas físicamente, hábito que les resta beneficios a su salud e incluso genera restricciones en esferas de la vida social, manejo del estrés, salud mental, estando más vulnerables a comorbilidades (Herazo et al., 2020; Caro \& Peñuela, 2017; González, Carreño, Estrada, Monsalve \& Álvarez, 2017; Suescún, et al., 2017). En este mismo sentido, también se ha mostrado que bajos niveles de AF se relacionan con una percepción de menor vitalidad por parte de los universitarios, asociando mayores niveles de actividad física con menor dolor corporal y mejor función física (De Mateo et al., 2018) por lo que se hace indispensable mantener dentro del currículo académico una formación dirigida a la importancia del cuidado de esta dimensión y en general de los hábitos y estilos de vida desde cualquier programa universitario.

Por su parte, al buscar posibles relaciones entre las dimensiones manejo del estrés y la actividad física, se observó que el comportamiento con tendencia a un mejor estilo de vida fue mayor en hombres que en mujeres; sin embargo, en las otras dimensiones evaluadas (nutrición, relaciones interpersonales, responsabilidad en salud y crecimiento espiritual) no se encontró diferencias significativas entre los sexos; lo anterior concuerda con Durán et al., (2017), quienes reportaron diferencias de los resultados al hacer la comparación entre sexos, pero contrastan en que fueron mejores los resultados para el componente de actividad física en el sexo masculino, situación que además difiere en el resto de las dimensiones, siendo los hombres el grupo con hábitos alimentarios inadecuados, mayor consumo de tabaco, alcohol y somnolencia diurna, al compararlos con los reportes de estilos de vida en el grupo de mujeres. No obstante, para Jiménez \& Ojeda (2017) no existió diferencias significativas a nivel general en la comparación de ambos sexos entorno a los estilos de vida en los sujetos observados; lo cual permite inferir que los hábitos y estilos de vida no están estricta y necesariamente relacionados con el sexo en las diferentes dimensiones.

El análisis individual de las dimensiones contempladas en el estudio no mostró diferencias concluyentes por estrato en los participantes, hallazgos que contrastan con la afirmación de Montenegro \& Ruiz (2019), los estratos 1, 2 y 3 tienen un estilo de vida clasificado como malo-regular en un (100\%) en comparación con los estratos 4, 5 y 6 con tan solo el (4,3\%) de clasificado con un estilo de vida muy malo-regular. Respecto a los hábitos de alimentación saludable en el presente estudio no fueron encontradas diferencias significativas entre los grupos analizados por estrato socioeconómico, aspecto que difiere con otro estudio, el cual evidenció que los estudiantes de estratos socioeconómicos bajos tienen mayor probabilidad de escaso consumo de frutas y verduras, así como elevado consumo de sal, grasas y azúcares Herazo et al. (2020). Lo anterior permite inferir que los estilos de vida más que relacionados con el estrato socioeconómico, puede estar ligado a la oportunidad de formación personal, familiar y cultural en que se desarrolla cada individuo.

Con respecto al análisis de las dimensiones estudiadas frente al sexo, al buscar posibles relaciones, no se encontraron diferencias significativas lo que se corresponde con lo reportado por Concha, Castillo, y Guzmán (2019) y Yaguachi, Reyes \& Poveda (2018); no obstante Lorenzini, Betancur, Chel, Segura \& Castellanos (2015), González et al. (2017) y Villaquirán et al., (2018) encontraron que existen diferencias entre sexos siendo los hombres reportados con mayor sobrepeso y obesidad y las mujeres 2,72 veces más sedentarias que los hombres. Por otra parte, se encontró que los participantes encuestados correspondiente al área de la salud no tienen hábitos saludables, lo cual se corrobora con otros estudios como el de Caro \& Peñuela (2017) y Chen, Rohaiza \& Soo (2018) en los cuales se muestra que a pesar de que las personas tengan conocimiento sobre 
salud alimentaria e incluso pertenezcan al área de la salud, sus hábitos no son saludables.

\section{Conclusión}

Los estudiantes encuestados reportaron un estilo de vida no saludable, siendo ello visible en los puntajes inferiores a los promedios definidos en la escala de evaluación, que permitió identificar el consumo de alimentos poco saludables, escasa regulación en el consumo de azúcares y grasas, así como una dieta baja en consumo de frutas y verduras, acompañado por insuficiente o ninguna dedicación a la práctica de actividad física, acorde con las recomendaciones emitidas por organismos de salud internacionales. El sexo y el estrato se constituyen en un factor explicativo del estilo de vida general de los estudiantes universitarios, los hombres son más activos físicamente y manejan el estrés en mayor medida que las mujeres. En relación con el estatus socioeconómico, se reportan mayores promedios en los estratos más altos en el puntaje general del cuestionario y en los niveles de actividad física. Los hábitos inadecuados de vida se observaron incluso en los estudiantes de las ciencias de la salud. La principal limitación de este estudio es su naturaleza transversal que no permite establecer relaciones causales entre las variables estudiadas.

\section{Recomendaciones}

El presente estudio es una aproximación inicial a la problemática de una población definida en una IES de la ciudad de Barranquilla, Colombia, el cual hizo parte de un estudio realizado en varias localidades de la Región Caribe del país. Dada las condiciones identificadas en los jóvenes participantes es necesario profundizar en los factores y sus causas, para proponer modelos multimodales de intervención efectivos mediante estrategias de información, comunicación y educación, apoyados en la participación del ente gubernamental, la familia y el mismo estudiante a fin de generar impacto positivo en el estado actual y futuro de los universitarios, al fomentar y concientizar en ellos hábitos de vida saludables. Lo anterior entendiendo que la detección de estilos de vidas en los jóvenes en etapa universitaria, favorece el establecimiento de medidas tempranas de promoción y prevención que incidan sobre las repercusiones que tienen estos hábitos, en el mantenimiento de la salud en la etapas próximas de la vida como lo son la adultez y la vejez, y así evitar el advenimiento de enfermedades que se relacionan directamente con los malos hábitos nutricionales y la falta de práctica de actividad física, para el desarrollo temprano de ENT.

Es importante considerar incluir en el plan de estudio de todos los programas universitarios asignaturas transversales que busquen sensibilizar y desarrollar conciencia en esta población respecto a los beneficios de adoptar hábitos saludables en la vida, para mantener y mejorar la condición de salud en general, con el apoyo de profesionales con habilidades afines a cada dimensión contemplada para lograr el cambio de comportamiento a uno favorable para la salud.

\section{Agradecimientos}

Al Departamento Administrativo de Ciencia, Tecnología e Innovación (Colciencias), por la financiación para el recurso humano que se requirió para el desarrollo del macro proyecto mediante la convocatoria 766 de 2018, contrato FP44842-305-2018. A la Universidad Simón Bolívar sede Barranquilla, Colombia, por su apoyo y respaldo institucional para el desarrollo del estudio.

\section{Referencias}

Bennasar M, et al. (2020) Cluster Analysis of HealthRelated Lifestyles in University Students. Int J Environ Res Public Health, 17(5), 1776. Recuperado de: https: / www.ncbi.nlm.nih.gov/pmc/articles/ PMC7084566/

Canova C. (2017). Estilo de vida de estudiantes universitarios de enfermería de Santa Marta, Colombia. Revista Colombiana De Enfermería, 14, 23-32. Recuperado de: https:// revistacolombianadeenfermeria.unbosque.edu.co/ article/view/2025

Canova C, Quintana M y Álvarez L. (2018). Estilos de Vida y su implicación en la salud de los estudiantes Universitarios de las Ciencias de la Salud: Una revisión sistemática. Revista Científica. 23(2). Recuperado de: https: / / publicacionescientificas.uces.edu.ar/ index.php/cientifica/article/view/531

Caro A, y Peñuela M. (2017). Factores individuales y socio-ambientales relacionados a la actividad física en universitarios de una institución de Barranquilla 2017. Universidad Del Norte. Recuperado de: http: / / manglar.uninorte.edu.co/handle/10584/ 7886? show=full

Chau C, y Tavera M. (2020). Estudio longitudinal de estilos de vida en estudiantes de una universidad pri- 
vada de Lima Metropolitana. Psicologia y Salud. 30(2), 253-263. Recuperado de: https:// psicologiaysalud.uv.mx/index.php/psicysalud/ article/view/2659

Chen T, Rohaiza S y Soo D. (2018). Dietary Habits and Lifestyle Practices among University Students in Universiti Brunei Darussalam. Malaysian Journal of Medical Sciences. 25(3), 56-66. Recuperado de: https:/ /www.ncbi.nlm.nih.gov/pmc/articles/ PMC6422551/

Concha Y, Castillo M, y Guzmán E. (2019). Comparación De La Calidad DeVida En Estudiantes Universitarios Según Nivel De Actividad Física. Universidad Y Salud. 22(1), 33-40. Recuperado de: https:// revistas.udenar.edu.co/index.php/usalud/article/ view $/ 4400$

Departamento Administrativo Nacional de Estadística DANE. Estratificación socioeconómica para servicios públicos domiciliarios. Recuperado de: https:/ /www.dane.gov.co/index.php/servicios-al-ciudadano/servicios-informacion/estratificacionsocioeconomica\#preguntas-frecuentes

De Mateo B, Camina M, Cartujo A, Carreño L, De la Cruz S y Redondo P. (2019). Health Perception According to the Lifestyle of University Students. J Community Health. 44, 74-80. Recuperado de: https:/ /link.springer.com/article/10.1007/s10900-0180555-4

De Souza M, Zea A, Rodríguez G y Molina A. (2017). Estilos de vida y factores socioeconómicos en estudiantes de electiva de actividad física y deporte de la Pontificia Universidad Javeriana. Análisis. 49(90). Recuperado de: https://dialnet.unirioja.es/servlet/ articulo? codigo $=6140729$

Durán S, Crovetto M, Espinoza V, Mena F, Oñate G, Fernández M, ..., Valladares M. (2017) Lifestyles, body mass index and sleep patterns among university students. Rev Med Chil. 145(11), 1403-1411. Recuperado de: https://pubmed.ncbi.nlm.nih.gov/ 29664522/

Espinoza M, Vanegas J. (2017). Una revisión de los estilos de vida de estudiantes universitarios. Revista Torreón Universitario. 6(16). Recuperado de: https:// www.lamjol.info/index.php/torreon/article/ view/6554

Espinoza Lara ML. (2018) Validación de un cuestionario para medir el estilo de vida de los estudiantes Universitarios en el marco de la teoría de Nola Pender (EVEU).Torreon, 7(19, 38-9. Recuperado de: https:/ /www.camjol.info/index.php/torreon/article/ view/7909

Gómez Z, Landeros P, Romero E, y Troyo R. (2016). Estilos de vida y riesgos para la salud en una población universitaria. Revista de Salud Pública y Nutrición. 15 (2). Recuperado de: https:// www. medigraphic.com / c gi - bin / new / resumen. cgi? IDARTICULO $=67869$

González L, Carreño C, Estrada A, Monsalve J, y Álvarez L. (2017). Exceso De Peso Corporal En Estudiantes Universitarios Según Variables Sociodemográficas Y Estilos De Vida. Revista Chilena De Nutrición. 44(3), 251-61. Recuperado de: https://scielo.conicyt.cl/ scielo.php?script $=$ sci_arttext\&pid $=$ S071775182017000300251

Gore M, Menon K, Safai A, Shukla S y Yeravdekar R. (2020). Determinants of health-promoting lifestyles amongst Indian University students, International Journal of Health Promotion and Education. Recuperado de: https://www.tandfonline.com/doi/abs/ 10.1080/14635240.2020.1726202

Herazo Y, Nuñez N, Sánchez L, Vásquez F, Lozano A, Torres E, y Valdelamar A. (2020). Estilos de vida relacionados con la salud en estudiantes universitarios. Retos, 38, 547-551. Recuperado de: https:// recyt.fecyt.es/index.php/retos/article/view/ 72871

Jiménez O, y Ojeda R. (2017). Estudiantes universitarios y estilos de vida. Revista Iberoamericana de Producción Académica y Gestión Educativa, 4 (8). Recuperado de: https://www.pag.org.mx/index.php/PAG/ article/view/723

Lorenzini R, Betancur D, Chel L, Segura M, y Castellanos A. (2015). Estado Nutricional En Relación Con El Estilo De Vida De Estudiantes Universitarios Mexicanos. Nutrición Hospitalaria: Órgano Oficial De La Sociedad Española De Nutrición ParenteralY Enteral, 32(1), 94-100. Recupertado de: http://

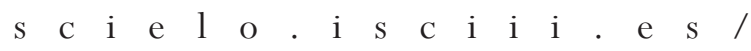
scielo.php?script $=$ sci_arttext\&pid $=$ S02 12 16112015000700015

Montenegro, A., y Ruíz, A. (2019). Factores asociados a los estilos de vida en los estudiantes universitarios. Una aplicación del instrumento fantástico. R. Actividad fis. y deporte, 6(1), 87-108. Recuperado de: https:/ /revistas.udca.edu.co/index.php/rdafd/article/ view/1432

Ministerio de Salud y Protección Social-MSPS. Encuesta nacional de la situación nutricional-ENSIN 2015. Recuperado de: https://www.minsalud.gov.co/ sites/rid/Lists/BibliotecaDigital/RIDE/VS/ED/ 
GCFI/documento-metodologico-ensin-2015.pdf

Oliveros N, Benitez J, García A \& Bello C. (2016). Estilos de vida en estudiantes de una universidad pública. CatedraVillarreal, 4(1). Recuperado de: http: / / revistas.unfv.edu.pe/index.php/ $\mathrm{RCV} /$ article/ view/63/63

Organización Mundial de la Salud. Enfermedades no trasmisibles. (2018). Recuperado de: https:// www.who.int/es/news-room/fact-sheets/detail/ noncommunicable-diseases

Pérez I, Rivera E, y Delgado M. (2017). Mejora de hábitos de vida saludables en alumnos universitarios mediante una propuesta de gamificación. Nutr Hosp, 34(4), 942-951. Recuperado de: https:// www.redalyc.org/pdf/3092/309252410040.pdf

Setia M.S. (2016). Methodology Series Module 3: Crosssectional Studies. Indian journal of dermatology, 61(3), 261-264. Recuperado de https: / / doi.org/10.4103/ 0019-5154.182410

Suescún S, et al. (2017). Estilos de vida en estudiantes de una universidad de Boyacá, Colombia. Rev. Fac. Med, 65(2), 227-31. Recuperado de: https:// revistas.unal.edu.co/index.php/revfacmed/ article/view/ 58640

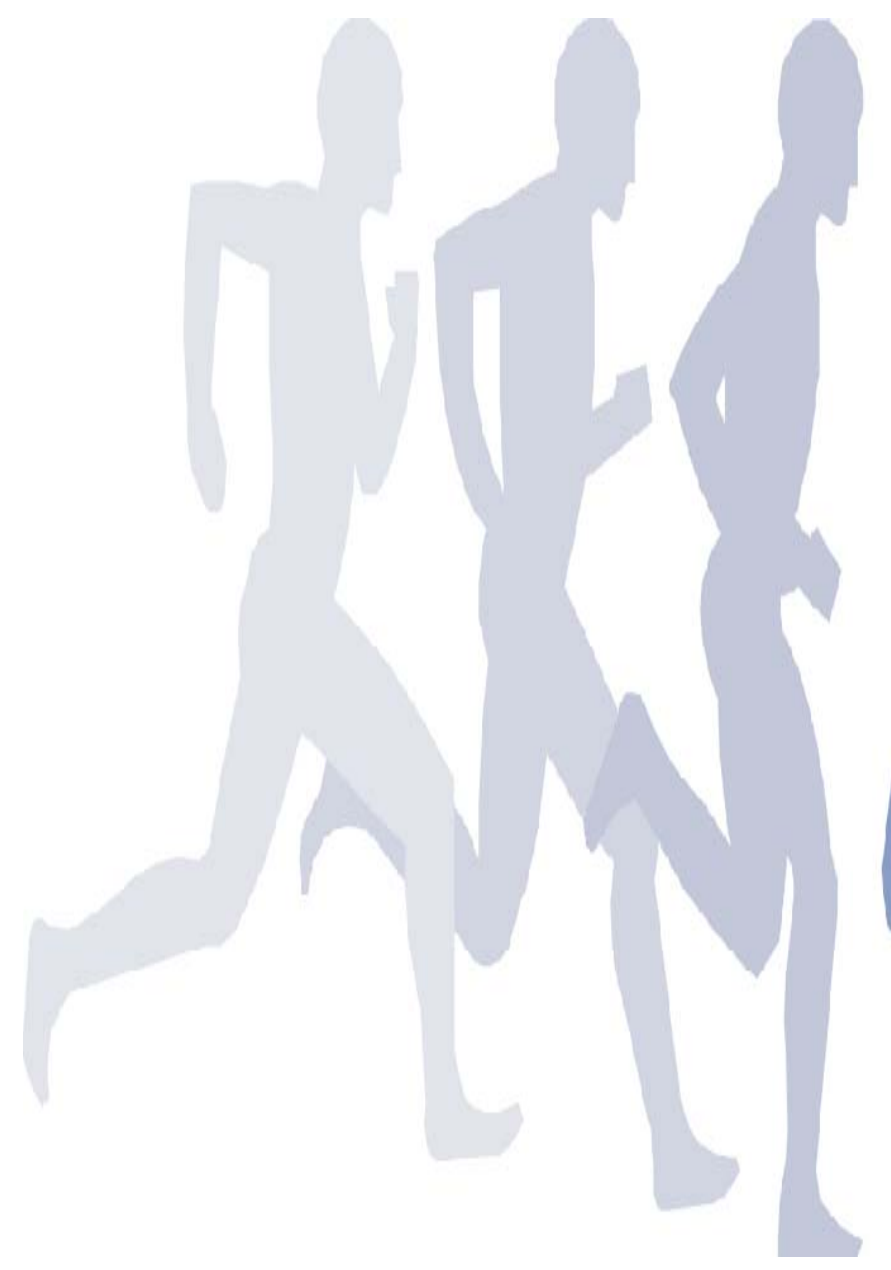

Varela M, Ochoa A, Tovar R. (2016). Tipologías de estilos de vida en jóvenes universitarios. Univ. Salud, 18(2), 246-256. Recuperado de: http:// www.scielo.org.co/pdf/reus/v18n2/v18n2a06.pdf Varmaghani M, Mansouri M, Shams M, Molla B, Saiyarsarai P, Emamikhah M, ..., Sharifit F. (2020). The relationship between lifestyle and anthropometric factors with the sleep characteristics among university students in Iran: the MEPHASOUS study. J Diabetes Metab Disord, 03-05. Recuperado de: https: / / link.springer.com/article/10.1007/ s40200-020-00598-x

Villaquirán A, Velasco J, Jimena S, y Ortega E. (2018). Factores Comportamentales Para Enfermedades No Transmisibles En Estudiantes Universitarios. Revista Ciencia Y Cuidado, 15(2), 52-64. Recuperado de: https : / / revistas.ufps.edu.co/index.php/ cienciaycuidado/article/view/1401

Yaguachi R, Reyes M, y Poveda C. (2018). Influencia De Estilos De Vida En El Estado Nutricional De Estudiantes Universitarios. Perspectivas En Nutricion $\mathrm{Hu}$ mana, 20(2), 145-56. Recuperado de: http:// www. scielo.org.co/scielo.php?pid =S0124$41082018000200145 \&$ script $={ }_{\text {sci_abstract } \& \text { tlng }}=\mathrm{es}$

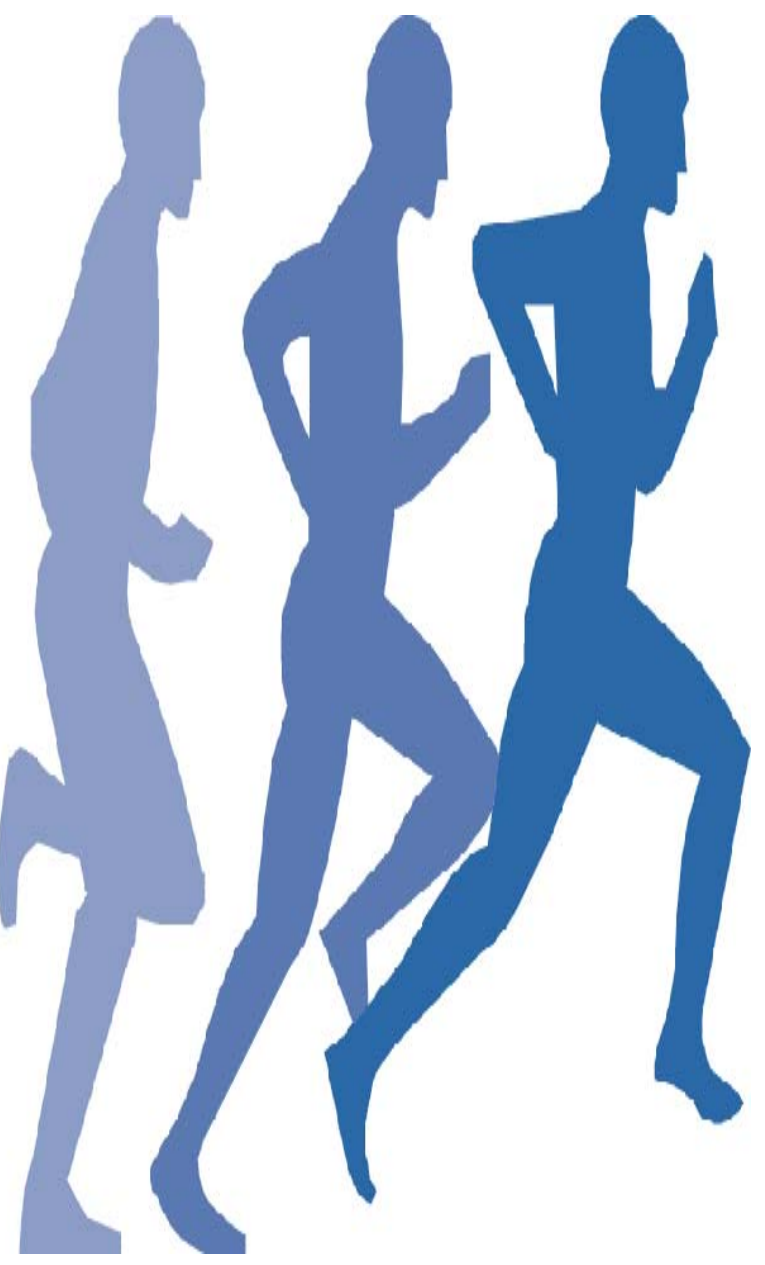

